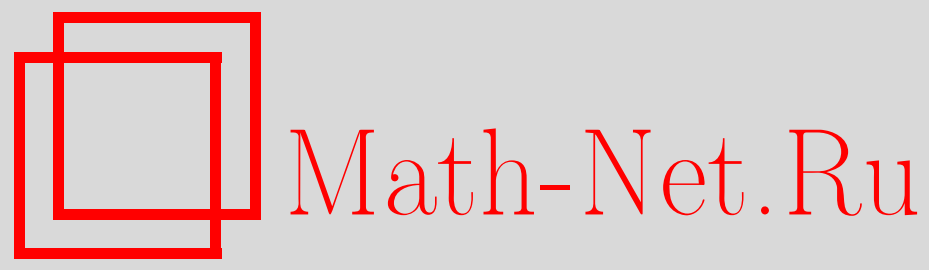

А. Н. Варченко, Д. Фелдер, Алгебраическая интегрируемость двухчастичного оператора Рюэйсенарса, Функи. анализ и его прил., 1998, том 32, выпуск 2, 8-25

DOI: https://doi.org/10.4213/faa410

Использование Общероссийского математического портала MathNet.Ru подразумевает, что вы прочитали и согласны с пользовательским соглашением http://www . mathnet.ru/rus/agreement

Параметры загрузки:

IP: 34.227 .88 .159

26 апреля 2023 г., 16:29:11

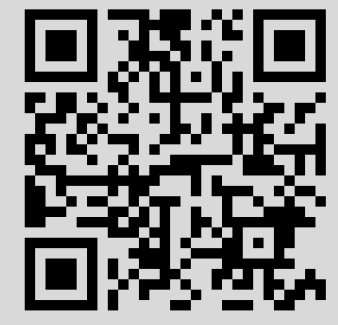


Функииональньй анализ и еәо приложения

1998, т. 32, вып. 2, с. 8-25

УДК 517.9

\title{
Алгебраическая интегрируемость двухчастичного оператора Рюэйсенарса
}

\author{
(C) 1998. А. Н. ВАРЧЕНко, Дж. ФЕЛДЕР
}

\section{§1. Введение}

Мы изучаем задачу на собственные значения $L \psi=\varepsilon \psi$ для разностного $q$-оператора Ламе $L$ одной комплексной переменной $\lambda$ :

$$
L \psi(\lambda)=\frac{\theta(\lambda-\gamma m, \tau)}{\theta(\lambda, \tau)} \psi(\lambda+\gamma)+\frac{\theta(\lambda+\gamma m, \tau)}{\theta(\lambda, \tau)} \psi(\lambda-\gamma) .
$$

Здесь $\gamma$ - комплексный параметр, $\tau$ - параметр, значения которого лежат в верхней полуплоскости, и $m$ - неотрицательное целое число.

Присутствующая в определении оператора $L$ тета-функция - это $\theta_{1}$-функция Якоби

$$
\theta(z, \tau)=-\sum_{j \in \mathbb{Z}+\frac{1}{2}} e^{\pi i j^{2} \tau+2 \pi i j\left(z+\frac{1}{2}\right)} .
$$

Разностный оператор $L$ возник в работе Склянина [10], посвященной теории представлений эллиптических алгебр Склянина. В самом деле, если мы заменим тета-функции в числителях тремя другими функциями Якоби $\theta_{\alpha}$, то получим разностные операторы, которые вместе с оператором $L$ будут удовлетворять коммутационным соотношениям алгебры Склянина.

Оператор $L$ появляется также как простейший нетривиальный квантовый релятивистский гамильтониан Калоджеро-Мозера, введенный Рюэйсенарсом [9]: $L$ сопряжен с двухчастичным гамильтонианом Рюэйсенарса, а его полуклассический (или нерелятивистский) вариант - это дифференциальный оператор Ламе. Более точно, $L=2 \mathrm{Id}+\operatorname{const} \gamma^{2} \ell+O\left(\gamma^{4}\right)$ при $\gamma \rightarrow 0$ и $\ell$ сопряжен с

$$
-\frac{d^{2}}{d \lambda^{2}}+m(m+1) \wp(\lambda)+\text { const }
$$

дифференциальным оператором Ламе из классической теории эллипсоидальных гармонических функций (см. [12]). Здесь ю обозначает двоякопериодическую функцию Вейерштрасса с периодами 1 и $\tau$.

Задача на собственные значения для оператора Ламе изучалась в классической литературе. В частности, Эрмит привел формулу для собственных

* Исследования первого автора выполнены при частичной поддержке NSF, грант DMS9501290 . 
функций этого оператора, которую мы сегодня могли бы назвать формулой типа «анзатца Бете» [12]: он выписал простую функцию, зависящую от $m$ параметров, и доказал, что эта функция будет собственной функцией оператора Ламе, если указанные параметры являются решениями некоторой системы $m-1$ алгебраических уравнений (или $m$ уравнений, если мы хотим задать собственное значение). Он также показал, что собственные функции, отвечающие общим собственным значениям, представляются в виде линейных комбинаций собственных функций, полученных таким образом.

В более современной терминологии можно сказать, что оператор Ламе является примером алгебраически интегрируемого, или конечнозонного, оператора Шрёдингера. Смысл этого утверждения проясняет следующая теорема, которая вытекает из классических результатов (но истинного автора которой, по-видимому, трудно установить); см. [3, 2].

Теорема 1.1. Существует дифференииальный оператор $\ell_{2 m+1}$ порядка $2 m+1$, такой, что алгебра $A$ дифференииальных операторов с мероморфньми коэффиииентами, коммутируюиих с $\ell$, порождается операторами $\ell$ $u \ell_{2 m+1}$. Кроме того, $A$ коммутативна $и$ отображение $\ell \mapsto x, \ell_{2 m+1} \mapsto y$ определяет изоморфизм алгебры $А$ на $\mathbb{C}[x, y] /\left(y^{2}-p(x)\right) \mathbb{C}[x, y]$ для некоторого полинома $p$ степени $2 m+1$.

Иными словами, алгебра дифференциальных операторов, коммутирующих с оператором Ламе, изоморфна алгебре полиномиальных функций на некоторой аффинной гиперэллиптической кривой. Эта кривая называется спектральной кривой дифференциального оператора $\ell$. Результаты Эрмита можно переформулировать следующим образом: спектральная кривая бирационально эквивалентна подмногообразию $m$-й симметрической степени эллиптической кривой $E_{\tau}=\mathbb{C} / \mathbb{Z}+\tau \mathbb{Z}$.

В [7] Кричевер и Забродин исследовали оператор $L$ и доказали его алгебраическую интегрируемость и конечнозонность. Они показали, что собственные функции оператора $L$ параметризуются точками некоторой гиперэллиптической кривой.

В [4] мы показали, что $L$ пропорционален трансфер-матрице неприводимого $(2 m+1)$-мерного представления эллиптической квантовой группы $E_{\tau, \gamma / 2}\left(s l_{2}\right)$. Анзатц Бете для этой трансфер-матрицы позволяет перенести результаты Эрмита на случай разностных операторов (см. теорему 3.1 из [4]) и дает формулу для собственных функций оператора $L$, параметризованных некоторой кривой Эрмита-Бете $Y$, заданной $m-1$ уравнениями от $m$ переменных на эллиптической кривой $E_{\tau}$. Мы напоминаем эти результаты в $\S 3$. В формулировке из [7] можно сказать, что мы описываем двойные функции Блоха через их нули. Результаты из [7] и [4] дополняют друг друга: «аддитивная» формула для собственных функций приведена в [7], а «мультипликативная» — в [4]. В обоих случаях формула зависит от параметров на гиперэллиптической кривой.

В этой статье мы изучаем разностные операторы, коммутирующие с $L$. Мы доказываем следующий результат. Пусть $D_{\gamma}$ - алгебра разностных опе- 
раторов вида

$$
M \psi(\lambda)=\sum_{j=a}^{b} B_{j}(\lambda) \psi(\lambda+j \gamma), \quad a \leqslant b \in \mathbb{Z}_{s}
$$

с 1-периодическими мероморфными коэффициентами $B_{j}$. Если $B_{a}$ и $B_{b}$ не равны нулю, то мы скажем, что $M$ имеет сmепень $b$ и длину $b-a$. Степень и длина произведения являются суммой степеней и длин сомножителей соответственно.

ТеОРема 1.2. Пусть $\gamma$ - иррачиональное число. Тогда существует разностныи оператор $N$ степени $2 m+1$ и длинь $4 m+2$, такой, что алгебра $A$ операторов из $D_{\gamma}$, коммутируюших с $L$, порождается операторами $L$ u $N$. Кроме того, А коммутативна и отображение $L \mapsto X, N \mapsto Y$ определяет изоморфизм из $A$ на $\mathbb{C}[X, Y] /\left(Y^{2}-P\left(X^{2}\right)\right) \mathbb{C}[X, Y]$ для некоторого полинома $P$ степени $2 m+1$.

В случае разностного оператора спектральная кривая имеет два инволютивных автоморфизма. Автоморфизм $(X, Y) \mapsto(X,-Y)$ соответствует симметрии $S \psi(\lambda)=\psi(-\lambda)$ задачи на собственные значения, как и в случае дифференциального оператора. Автоморфизм $(X, Y) \mapsto(-X,-Y)$ соответствует инволюции $U S \psi(\lambda)=e^{\pi i \lambda / \gamma} \psi(-\lambda)$, которая переводит собственные функции в собственные функции с противоположным собственным значением.

Теорему 1.2 можно вывести из результатов Кричевера и Забродина [7], используя [5] и [8]. Ниже мы приведем более прямое доказательство этой теоремы, которое также дает эффективную формулу для оператора $N$ и на самом деле для линейного базиса алгебры $A$. Действительно, оператор $N$ можно явно выразить через замечательное семейство разностных операторов $M_{l}$, коммутирующих с $L$ :

Теорема 1.3. Пусть $\gamma \neq 0 \bmod \mathbb{Z}+\tau \mathbb{Z}$. Для каждого общего $l \in \mathbb{C}$ существует коммутирующий с $L$ разностный оператор $M_{l}$ вида

$$
M_{l} \psi(\lambda)=\sum_{k=0}^{m} A_{l-m+2 k}^{l}(\lambda / \gamma) \psi(\lambda+(l-m+2 k) \gamma),
$$

где коэффициенть $A_{l-m+2 k}^{l}$ задаются с помощью тета-функций Якоби (см. формулу (3)). Эти операторы попарно коммутируют и удовлетворяют соотношениям

$$
L M_{l}=\frac{\theta(\gamma(l-m), \tau)}{\theta(\gamma l, \tau)} M_{l+1}+\frac{\theta(\gamma(l+m), \tau)}{\theta(\gamma l, \tau)} M_{l-1},
$$

и правилам умножения

$$
M_{l} M_{k}=\sum_{j} A_{j}^{l}(k) M_{k+j} .
$$

Кроме того, $L$ пропорчионален $M_{m-1}$, a $N$ пропорчионален $M_{m+1}-M_{-m-1}$. 
Таким образом, мы можем сказать, что, с точностью до замены переменных, $M_{l}$ является операторнозначной собственной функцией оператора Ламе с «собственным значением» $L$. Конструкция этих операторов описана в $\S 4$.

В §5 мы вычисляем собственные значения оператора $M_{l}$ на наших собственных функциях через параметры анзатца Бете. Эти собственные значения являются функциями на гиперэллиптической кривой с особенностями на бесконечности. Это позволяет нам установить связь с конструкцией Кричевера (для вырожденного случая) [5], в которой изоморфизм между алгеброй $A$ разностных операторов, коммутирующих с $L$, и алгеброй функций на гиперэллиптической кривой задается с помощью этого отображения взятия собственного значения. В частности, если $l$ - целое число, то собственное значение оператора $M_{l}$ - это мероморфная функция на гиперэллиптической кривой, однозначно определенная своими полюсами и нулями на бесконечности. Из этого замечания следует, что образы операторов $M_{l}$ с целыми $l$ связаны с базисом функций, определенным Кричевером и Новиковым в [6].

Операторы $M_{l}$ определяют на алгебре разностных операторов, коммутирующих с $L$, структуру обобщенно-градуированной алгебры; определения cм. B $[6]$.

Задача на собственные значения $L \psi=\varepsilon \psi$ превращается после замены зависимой переменной $\psi(\lambda)=u(\lambda) \prod_{j=1}^{m} \theta(\lambda-j \gamma)$ в

$$
u(\lambda+\gamma)+\frac{\theta(\lambda+\gamma m, \tau) \theta(\lambda-\gamma(m+1), \tau)}{\theta(\lambda, \tau) \theta(\lambda-\gamma, \tau)} u(\lambda-\gamma)=\varepsilon u(\lambda)
$$

Это уравнение имеет эллиптические коэффициенты. Отметим, кстати, что если $\gamma \in \mathbb{Z}+\tau \mathbb{Z}$, то это уравнение с постоянными коэффициентами и легко получить его решение в терминах экспоненциальных функций.

В последнем параграфе настоящей статьи мы вычислим разностную групny Галуа (аналог дифференциальной группы Галуа для дифференциальных уравнений) этого уравнения в случае иррационального $\gamma$. Это есть группа Галуа расширения разностного поля эллиптических функций, порожденного решениями рассматриваемого уравнения. Оказывается, что она является абелевой группой, что согласуется с концепцией работы [1], где алгебраически интегрируемые дифференциальные операторы характеризуются абелевостью соответствующих дифференциальных групп Галуа.

Мы благодарны Игорю Кричеверу за ряд интересных и полезных замечаний к первому варианту этой статьи.

\section{§2. Обозначения для эллиптических чисел}

Мы предполагаем, что $\gamma \neq 0 \bmod \mathbb{Z}+\tau \mathbb{Z}$. Удобно ввести новую переменную $x=\lambda / \gamma$. Относительно этой переменной шаг разностного оператора $L$ является целым числом, а его периоды - это $\omega=\gamma^{-1}$ и $\omega^{\prime}=\tau \gamma^{-1}$. Эллиnтическое число

$$
[x]=\frac{\theta(\gamma x, \tau)}{\theta(\gamma, \tau)}
$$


- это целая нечетная функция от $x$ с нулями на решетке $\mathbb{Z} \omega+\mathbb{Z} \omega^{\prime}$, обладающая следующими трансформационными свойствами:

$$
[x+\omega]=-[x], \quad\left[x+\omega^{\prime}\right]=-e^{-\pi i \tau-2 \pi i \gamma x}[x]=-e^{-\frac{\pi i}{\omega}\left(\omega^{\prime}+2 x\right)}[x] .
$$

Относительно этой переменной, которую мы будем использовать до конца статьи, $q$-оператор Ламе принимает вид

$$
L=\frac{[x-m]}{[x]} T_{1}+\frac{[x+m]}{[x]} T_{-1}, \quad T_{j} \psi(x)=\psi(x+j) .
$$

Удобно также ввести эллиптические биномиальные коэффициенты и эллиптические факториаль:

$$
\left[\begin{array}{l}
x \\
n
\end{array}\right]=\frac{[x][x-1] \cdots[x-n+1]}{[n][n-1] \cdots[1]}, \quad\left[\begin{array}{l}
x \\
0
\end{array}\right]=1, \quad[n] !=[1][2] \cdots[n] .
$$

\section{§3. Собственные функции Бете}

Напомним результаты из [4] относительно q-оператора Ламе. Мы предполагаем, что $\gamma \neq 0 \bmod \mathbb{Z}+\tau \mathbb{Z}$. Введем $m$ функций от $t=\left(t_{1}, \ldots, t_{m}\right) \in \mathbb{C}^{m}$

$$
b_{i}(t)=\frac{\left[t_{i}-m\right]}{\left[t_{i}+m\right]} \prod_{j: j \neq i} \frac{\left[t_{j}-t_{i}-1\right]}{\left[t_{j}-t_{i}+1\right]}, \quad i=1, \ldots, m .
$$

Теорема 3.1 [4]. Пусть $\left(t_{1}, \ldots, t_{m}, c\right)$ - решение уравнений анзатца Бeme

$$
b_{i}(t)=e^{2 \gamma c}, \quad i=1, \ldots, m,
$$

maкое, ито $t_{i} \neq t_{j} \bmod \omega \mathbb{Z}+\omega^{\prime} \mathbb{Z}$, если $i \neq j$. Тогда

$$
\psi(x)=e^{c \gamma x} \prod_{j=1}^{m}\left[x+t_{j}\right]
$$

является решением q-уравнения Ламе

$$
\frac{[x-m]}{[x]} \psi(x+1)+\frac{[x+m]}{[x]} \psi(x-1)=\varepsilon_{L} \psi(x),
$$

с собственным значением

$$
\varepsilon_{L}(t)=e^{-\gamma c} \frac{[2 m]}{[m]} \prod_{j=1}^{m} \frac{\left[t_{j}+m-1\right]}{\left[t_{j}+m\right]} .
$$

ЗАмеЧАниЕ. Переменной $t_{j}$ из [4] здесь соответствует $\left(t_{j}-1 / 2\right) \gamma$. Кроме того, $\gamma=2 \eta$ в обозначениях из [4].

Отметим, что функции $b_{j}$ удовлетворяют условиям

$$
\begin{aligned}
b_{j}\left(t_{1}, \ldots, t_{i}+\omega, \ldots, t_{m}\right) & =b_{j}\left(t_{1}, \ldots, t_{i}, \ldots, t_{m}\right), \\
b_{j}\left(t_{1}, \ldots, t_{i}+\omega^{\prime}, \ldots, t_{m}\right) & =e^{4 \pi i \gamma} b_{j}\left(t_{1}, \ldots, t_{i}, \ldots, t_{m}\right) .
\end{aligned}
$$


для всех $i, j$. Таким образом, если $\left(t_{1}, \ldots, t_{m}, c\right)$ - некоторое решение, то $\left(t_{1}, \ldots, t_{i}+\omega, \ldots, c\right)$ и $\left(t_{1}, \ldots, t_{i}+\omega^{\prime}, \ldots, c+2 \pi i\right)$ также являются решениями. Более того, у этих решений одинаковые собственные функции. Далее, и эти уравнения, и собственные функции симметричны относительно перестановок переменных $t_{j}$. На $\mathbb{C}^{m} \times \mathbb{C}$, таким образом, действует полупрямое произведение $\Gamma=\left(\omega \mathbb{Z}+\omega^{\prime} \mathbb{Z}\right)^{m} \widetilde{\times} S_{m}$, и уравнения анзатца Бете можно рассматривать на соответствуюшем факторе: множество решений - это

$$
\begin{aligned}
X_{\infty} & =\left\{(t, c) \in\left(\mathbb{C}^{m}-D\right) \times \mathbb{C} \mid b_{j}(t)=e^{2 \gamma c}, j=1, \ldots, m\right\} / \Gamma, \\
D & =\bigcup_{i<j}\left\{t_{i}=t_{j} \bmod \omega \mathbb{Z}+\omega^{\prime} \mathbb{Z}\right\} .
\end{aligned}
$$

На $X_{\infty}$ мы имеем $\mathbb{Z}$-действие $(t, c) \mapsto(t, c+\pi i / \gamma)$. Соответствующий фактор - это множество

$$
X=X_{\infty} / \mathbb{Z},
$$

определенное уравнениями $b_{i}(t)=b_{j}(t)$. Оно будет алгебраическим подмногообразием симметрической степени нашей эллиптической кривой. Собственное значение $\varepsilon_{L}$ является двузначной функцией на $X$. На двулистном накрытии

$$
X_{2}=X_{\infty} / 2 \mathbb{Z}
$$

оно становится однозначной мероморфной функцией. Собственные функции, отвечающие точкам из $X_{\infty}$, которые имеют одну и ту же проекцию на $X_{2}$, пропорциональны в том смысле, что получаются одна из другой умножением на некоторую 1-периодическую функцию.

Другими словами, точке из $X_{2}$ отвечает некоторое одномерное векторное пространство собственных функций над полем $K$ 1-периодических мероморфных функций. Отметим, что все эти функции соответствуют одному и тому же собственному значению.

Собственные функции, отвечающие точкам из $X_{2}$, которые имеют одну и ту же проекцию на $X$, переводятся друг в друга отображением $U: \psi \mapsto$ $e^{\pi i x} \psi$. Они имеют противоположные собственные значения.

В окрестности точки $c=\infty$ эти многообразия можно описать более явно:

Лемма 3.2. Пусть $\bar{X}$ - замыкание многообразия $X$ в симметрической степени $S^{m} E$ эллиптической кривой $E=\mathbb{C} / \omega \mathbb{Z}+\omega^{\prime} \mathbb{Z}$. Тогда $\bar{X}$ содержит две точки $P_{+}=(-m+1, \ldots,-1,0)$ и $P_{-}=(m-1, \ldots, 1,0)$. Неприводимая компонента (или компоненть) многообразия $X$, содержащая $P_{+} u P_{-}$, образует кривую $Y$, являюшуюся в этих точках гладкой. Относительно локальной координать с нулем в $P_{+}$собственное значение $\varepsilon_{L}$ имеет вид const $u^{-1 / 2}(1+O(u)) u e^{2 c \gamma}=$ const $u^{-1}+O(1)$.

Пусть $Y_{2} \subset X_{2}$ - двулистное накрытие кривой $Y$. Это кривая, на которой собственное значение является однозначной функцией. Точки $P_{+}, P_{-}$ связаны симметрией $(t, c) \mapsto(-t,-c)$ уравнений анзатца Бете.

Теорема 3.3. Пусть $\gamma \in \mathbb{C}$ находится в общем положении. Для каждого общего $\varepsilon \in \mathbb{C}$ в $X_{\infty}$ имеется ровно два решения

$$
\left(t_{1}, \ldots, t_{m}, c\right) \quad u \quad\left(-t_{1}, \ldots,-t_{m},-c\right),
$$


уравнений анзатиа Бете (1) с заданным собственным значением $\varepsilon_{L}=\varepsilon$. Соответствующие функции $\psi_{ \pm}$линейно независимь над полем $K$ 1-периодических мероморфных функиий от $x$, и все решения q-уравнения Ламе $L \psi=$ $\varepsilon_{L} \psi$ являются линейныли комбиначиями функций $\psi_{+}, \psi_{-}$с коэффичиентами из $K$.

ОПРЕДЕЛЕНИЕ. Многозначная функция на $X \times \mathbb{C}$

$$
\psi(t, x)=e^{c \gamma x} \prod_{j=1}^{m} \frac{\left[x+t_{j}\right]}{\left[t_{j}\right]},
$$

где $c$ определяется с помощью уравнений анзатца Бете, называется функиией Бейкера-Ахиезера.

Функция Бейкера-Ахиезера однозначна на $X_{\infty} \times \mathbb{C}$.

Лемма 3.4. Если $M=\sum_{j} A_{j}(x) T_{j}$ - разностньй оператор с конечньлм иислом ненулевых коэффичиентов $A_{j}$, такой, что $M \psi(t, \cdot)=0$ для всех точек $t$ кривой $Y$, то $M=0$.

ДокаЗАтельство. Рассмотрим уравнение $M \psi(t, x)=0$ в окрестности точки $P_{+}$из леммы 3.2 . Пусть $k$ - наибольшее число, такое, что $A_{k} \neq 0$. Поскольку $\psi(t, x+j) / \psi(t, x)$ ведет себя как $e^{\gamma c j} \sim u^{-j / 2}$ при $u \rightarrow 0$, для того чтобы $M \psi / \psi$ было равно нулю, необходимо, чтобы старший коэффициент $A_{k}(x)$ был равен нулю, что противоречит предположению. Таким образом, $M=0$.

\section{§4. Разностные операторы, коммутирующие с $q$-оператором Ламе}

Построим последовательность разностных операторов, коммутирующих с $q$-оператором Ламе $L$. Пусть

$$
M_{l}=A_{l-m}^{l}(x) T_{l-m}+A_{l-m+2}^{l}(x) T_{l-m+2}+\cdots+A_{l+m}^{l}(x) T_{l+m} .
$$

Обычно $l$ - целое число, но иногда мы будем брать в качестве $l$ произвольное комплексное число. Для $k=0, \ldots, m$

$$
A_{l-m+2 k}^{l}(x)=(-1)^{k}\left[\begin{array}{c}
m \\
k
\end{array}\right] \prod_{j=0}^{m-k-1} \frac{[l+m-j][x+m-j]}{[x+l+k-j]} \prod_{j=0}^{k-1} \frac{[l-m+j][x-m+j]}{[x+l-m+k+j]},
$$

причем произведение по пустому множеству индексов предполагается равным нулю.

TeOpema 4.1. (i) $M_{m}=\frac{[2 m] !}{[m] !} \mathrm{Id}, M_{m-1}=\frac{[2 m-1] !}{[m-1] !} L$.

(ii) $M_{l} M_{k}=M_{k} M_{l}$ для любых комплексньх чисел $l, k$.

ДокАЗАТЕльство. (i) Если $l=m$, то все коэффициенты $A_{l-m+2 k}^{l}$ с $k \neq 0$ равны нулю из-за сомножителя $[l-m]$ во втором произведении. Если $k=0$, TO

$$
A_{0}^{m}(x)=\left[\begin{array}{c}
m \\
0
\end{array}\right] \prod_{j=0}^{m-1}[2 m-j]=\frac{[2 m] !}{[m] !}
$$


Аналогично, если $l=m-1$, то единственными ненулевыми коэффициентами будут

$$
A_{1}^{l}(x)=[2 m-1] ![x-m] /[m-1] ![x]=A_{-1}^{l}(-x) .
$$

(ii) Прежде всего, покажем, что $M_{l}$ коммутирует с $q$-оператором Ламе $L$. Уравнение $M L=L M$ для оператора вида $M=\sum A_{j} T_{j}$ эквивалентно тождеству

$$
\begin{aligned}
A_{j}(x) & \frac{[x+j+m]}{[x+j]}+A_{j-2}(x) \frac{[x+j-2-m]}{[x+j-2]} \\
= & A_{j}(x-1) \frac{[x+m]}{[x]}+A_{j-2}(x+1) \frac{[x-m]}{[x]},
\end{aligned}
$$

для его коэффициентов. Мы должны показать, что наши $A_{j}^{l}$, среди которых ненулевыми будут только коэффициенты с индексами $j$, принадлежащими множеству $\{l-m, l-m+2, \ldots, l+m\}$, являются решениями этого уравнения. Если мы подставим соответствующие формулы в тождество, которое нужно доказать, и сократим общие множители, то получим

$$
\begin{gathered}
\frac{[l-m+k-1][x-m+k-1][x+l+2 k]}{[k][x+l+k]}-\frac{[l+k][x+k][x+l+2 k-2 m-2]}{[m-k+1][x+l+k-m-1]} \\
=\frac{[l-m+k-1][x+k][x-m-1][x+l+2 k-m-1]}{[k][x][x+l+k-m-1]} \\
-\frac{[l+k][x+m+1][x+k-m-1][x+l+2 k-m-1]}{[m-k+1][x][x+l+k]} .
\end{gathered}
$$

Воспользуемся свойствами эллиптических чисел относительно сдвигов на $\omega=\gamma^{-1}$ и $\omega^{\prime}=\gamma^{-1} \tau$. Разделив обе части этого уравнения на $[x+k]$, получаем уравнение, все члены которого являются периодическими функциями от $x$ с периодом $\omega$ и умножаются на $\exp (2 \pi i \gamma(m-k+1))$, когда $x$ заменяется на $x+\omega^{\prime}$. Полюсы (они являются простыми) расположены в точках $x=0,-l-k,-k,-l+m-k+1$. Однако разность между левой и правой частями, как нетрудно убедиться, имеет в этих полюсах нулевой вычет для любого общего значения $k$. Но если $\alpha$-общее комплексное число, то единственная целая голоморфная функция $f$, такая, что $f(z+\omega)=f(z)$ и $f\left(z+\omega^{\prime}\right)=\alpha f(z)$ - это $f=0$. Таким образом, наше тождество доказано для общих, а в силу аналитичности и для всех значений $k$.

Чтобы завершить доказательство перестановочности операторов $M_{l}$ и $L$, мы должны отдельно проверить указанное тождество для коэффициентов в двух крайних случаях $j=l-m$ и $j=l+m+2$, когда только два члена оказываются ненулевыми. Это сделать нетрудно.

Это завершает доказательство п. (ii), если $l=m-1$. Для доказательства общего случая заметим, что для любой точки $t \in X$

$$
L M_{l} \psi(t, \cdot)=M_{l} L \psi(t, \cdot)=\varepsilon_{L}(t) M_{l} \psi,(t, \cdot) .
$$

Следовательно, $M_{l} \psi(t, \cdot)$ - собственная функция оператора $L$ с собственным значением $\varepsilon_{L}(t)$. Согласно теореме 3.3 , она пропорциональна функции 
$\psi(t, \cdot)$. То же самое имеет место и для $M_{k}$. Значит, для общего $t$ имеет место равенство $\left[M_{l}, M_{k}\right] \psi(t, \cdot)=0$. Из леммы 3.4 вытекает соотношение $\left[M_{l}, M_{k}\right]=0$.

Лемма 4.2. Пусть операторы $S$ и $U$ задаются формулами $S \psi(x)=$ $\psi(-x)$ и $U \psi(x)=e^{\pi i x} \psi(x)$. Тогда $M_{l} S=S M_{-l}$ u $U M_{l}=e^{\pi i(l-m)} M_{l} U \partial \Omega \Omega$ вcex $l$.

ДоказАтЕЛЬство. Первое утверждение вытекает из соотношения $T_{j} S=$ $S T_{-j}$ и тождества

$$
A_{j}^{l}(x)=A_{-j}^{-l}(-x) .
$$

Второе следует из соотношения $U T_{l-m+2 k}=e^{\pi i(l-m)} T_{l-m+2 k} U$.

ЛЕмма 4.3. Пусть $\gamma$ - иррациональное число $u \omega=1 / \gamma$. Пусть $\phi(x)=$ $\prod_{k=1}^{m}[x-k]$. Тогда для любого $j \in \mathbb{C}$ первыи коэффичиент любого разностного оператора $M=\sum_{k=0}^{l} B_{j-k}(x) T_{j-k} \quad c \omega$-периодическими коэффичиентами $B_{j-k}$, коммутирующего с $L$, имеет вид $B_{j}(x)=c \phi(x) / \phi(x+j)$, где c - некоторая константа.

ДокАзАТЕльство. q-оператор Ламе $L$ можно записать в следующей форме:

$$
L=\phi(x) T_{1} \phi(x)^{-1}+\phi(-x) T_{-1} \phi(-x)^{-1} .
$$

Отсюда следует, что $M_{\phi}=\phi^{-1} M \phi$ коммутирует с $T_{1}+C(x) T_{-1}$ для некоторой функции $C$. Таким образом, коэффициент при $T_{j}$ в $M_{\phi}$ подчиняется разностному уравнению $T_{1} f(x)=f(x)$, или $f(x+1)=f(x)$. Но $f$ также является $\gamma^{-1}$-периодической, поскольку $M$ принадлежит $D_{\gamma}$. Если $\gamma$ иррационально, то $f$ - это константа $c$. Таким образом, $M=c \phi(x) T_{j} \phi(x)^{-1}+\cdots=$ $c \phi(x) \phi(x+j)^{-1} T_{j}+\cdots$.

ЛЕмма 4.4. Пусть ү иррачионально. Если $l \geqslant m+1$ или если $l \leqslant-m-1$, то $M_{l}$ имеет степень $l+m$ и длину $2 m$. Если $l \in\{-m,-m+1, \ldots, m\}$, то $M_{l}$ имеет степень $|m-l|$ и длину $2|m-l|$.

ДокаЗАТЕЛЬСтво. Это означает, что нужно выяснить, когда коэффициенты $A_{k}^{l}$ обращаются в нуль, а это несложно сделать, если дано их разложение.

ЛЕмма 4.5. Предположим, что $\gamma$ иррачионально, и пусть $\omega=1 / \gamma$. Предположим, что $j$ - или общее комплексное число, или отричательное челое число. Тогда любой коммутируюший $c$ L разностный оператор вида

$$
M=B_{j}(x) T_{j}+B_{j-2}(x) T_{j-2}+\cdots+B_{j-2 l}(x) T_{j-2 l}, \quad B_{j} \neq 0,
$$

c $\omega$-периодическими коэффичиентами $B_{l}$ имеет длину $\geqslant 2 m$.

ДокаЗАТЕльство. Пусть $M-$ ненулевой оператор длины $<2 m$. Предположения относительно $j$ гарантируют, что функция $A_{j-2 k}^{j-m-2 k}(x)$ не равна тождественно нулю при $k=0,1,2, \ldots$ Значит, мы можем вычесть из $M$ подходящую линейную комбинацию

$$
M_{c}=c_{0} M_{j-m}+c_{1} M_{j-m-2}+\cdots+c_{m-1} M_{j-m-2(m-1)}
$$


так, чтобы степень получившегося в результате полинома не превосходила $j-2 m$. Мы утверждаем, что $M=M_{c}$. Так как длина оператора $M_{c}$ больше или равна $2 m$, за исключением случая, когда все $c_{l}$ равны нулю (см. лемму 4.4), то отсюда будет следовать, что $M=0$, — противоречие. Чтобы доказать наше утверждение, предположим, что $M-M_{c}$ имеет степень $d \leqslant j-2 m$. Коэффициент при $T_{d}$ в $M-M_{c}$ должен иметь вид $a \phi(x) / \phi(x+d)$ для некоторой константы $a \neq 0$. В частности, он имеет полюс в $x=m-d$. С другой стороны, этот коэффициент равен

$$
-c_{0} A_{d}^{j-m}(x)-\cdots-c_{m-1} A_{d}^{j-3 m+2}(x),
$$

причем $M$ не вносит в него никакого вклада, так как имеет длину, меньшую $2 m$. Члены этой суммы имеют вид const $A_{d}^{l}(x)$, где $d \leqslant l+m-2$, т. е. $A_{l-m+2 k}^{l}(x)$, где $k \leqslant m-1$. Полюс функции $A_{l-m+2 k}^{l}$ с наибольшей вещественной частью - это $x=-l+m-k$, и соответствующая вещественная часть меньше, чем вещественная часть числа $m-d=-l+2 m-2 k$, если $k \leqslant m-1$. Значит, функция (4) регулярна в $x=m-d-$ противоречие.

ОПредЕлЕниЕ. Пусть $S$ - инволюция, определяемая формулой $S \psi(x)=$ $\psi(-x)$. Разностный оператор $M$ называется симметрическим, если $M S=$ $S M$. Он называется антисимметрическим, если $M S=-S M$.

Лемма 4.6. Пусть $\gamma$ иррационально. Тогда любой симметрический оператор из $D_{\gamma}$, которьй коммутирует $c L$, является полиномом от $L$.

ДокАЗАтЕльство. Предположим, что $M \in D_{\gamma}$ - симметрический разностный оператор степени $j$, который коммутирует с $L$. Проведем доказательство по индукции. Если $j=0$, то $M$ в силу леммы 4.3 равен тождественному оператору, умноженному на константу. Пусть $j>1$. Тогда коэффициент при $T_{j}$ имеет вид, указанный в лемме 4.3. Если мы вычтем $c_{j} L^{j}$ из $M$, то получим симметрический разностный оператор степени, не превосходящей $j-1$, который по предположению индукции является полиномом от $L$.

ТЕОремА 4.7. Если $\gamma$ иррачионально, то все операторы из $D_{\gamma}$, коммутируюшие с L, являютсл полиномами от $L$ и

$$
N=M_{m+1}-S M_{m+1} S \text {. }
$$

ДокАЗАТЕльство. Для симметрического оператора мы доказали это утверждение в лемме 4.6. Предположим, что $M \in D_{\gamma}$ - антисимметрический оператор, коммутирующий с $L$. Если $M$ имеет степень $d \geqslant 2 m+1$, то мы можем вычесть из него умноженный на константу оператор $N L^{d-2 m-1}$, который антисимметричен и имеет степень $d$ по лемме 4.4 , и получить оператор меньшей степени. Это возможно в силу леммы 4.3. Таким образом, мы можем предполагать, что $M$ - антисимметрический оператор степени $\leqslant 2 m$. Вычтя из $M$ подходящий полином от $L$ степени $\leqslant 2 m$, мы получаем оператор, коммутирующий с $L$, вида $M^{\prime}=B_{-1}(x) T_{-1}+\cdots+B_{-2 m}(x) T_{-2 m}$. Чтобы применить лемму 4.5 , мы запишем $M^{\prime}=M_{o}^{\prime}+M_{e}^{\prime}$, где $M_{o}^{\prime}=B_{-1} T_{-1}+$ 
$B_{-3} T_{-3}+\cdots$ является суммой нечетных членов. Тогда и $M_{o}^{\prime}$, и $M_{e}^{\prime}$ коммутируют с $L$, поскольку свойство коммутирования с $L$ эквивалентно соотношениям, включающим в себя только четные или только нечетные коэффициенты. Но оба оператора $M_{o}^{\prime}$ и $M_{e}^{\prime}$ имеют отрицательную степень и длину $<2 m$, а значит, равны нулю по лемме 4.5.

Таким образом, алгебра $A$ порождается операторами $L$ и $N$. Так как $N$ антисимметрический, его квадрат является симметрическим и в силу леммы 4.6 имеет место соотношение

$$
N^{2}=Q(L)
$$

для некоторого полинома $Q$. Сравнивая степени, мы убеждаемся, что $Q$ имеет степень $4 m+2$. Согласно лемме $4.2, N^{2}$ коммутирует с $U$, но $L U=$ $-U L$. Так как операторы $L^{j}$ линейно независимы (они имеют разные степени), то $Q(L)=P\left(L^{2}\right)$ для некоторого полинома $P$ степени $2 m+1$.

Теперь мы можем завершить доказательство теоремы 1.2. Тот факт, что $L$ и $N$ коммутируют и подчиняются указанному соотношению, означает, что отображение $X \mapsto L, Y \mapsto N$ определяет сюръективный гомоморфизм алгебр

$$
h: \mathbb{C}[X, Y] /\left(Y^{2}-P\left(X^{2}\right)\right) \mathbb{C}[X, Y] \rightarrow A .
$$

Любой элемент левой алгебры однозначно представляется полиномом вида $f(X)+g(X) Y$. Такой полином принадлежит ядру гомоморфизма $h$ тогда и только тогда, когда $f(L)+g(L) N$ равен нулю. Но это означает, что симметрическая и антисимметрическая части $f(L)$ и $g(L) N$ по отдельности равны нулю. Рассматривая коэффициент при $T_{j}$ с наибольшим $j$, мы с помощью индукции убеждаемся, что все коэффициенты полиномов $f$ и $g$ равны нулю. Таким образом, гомоморфизм $h$ является изоморфизмом.

\section{$\S 5$. Собственные значения}

В этом параграфе мы вычислим собственные значения наших коммутирующих операторов на функции Бейкера-Ахиезера $\psi(t, x)$. Это отображение взятия собственного значения переводит элемент алгебры коммутирующих разностных операторов (т. е. функцию на гиперэллиптической кривой) в двузначную функцию на кривой Эрмита-Бете $Y$ и, как будет далее показано, осуществляет бирациональную эквивалентность между этой гиперэллиптической кривой и двулистным накрытием кривой Эрмита-Бете.

Начнем с описания некоторых замечательных свойств разностных операторов $M_{l}, l \in \mathbb{C}$.

ПРЕДЛОЖЕНИЕ 5.1. Для любого общего комплексного иисла $l$

$$
L M_{l}=\frac{[l+m]}{[l]} M_{l-1}+\frac{[l-m]}{[l]} M_{l+1} .
$$

ДокАЗАТЕЛЬСтво. Если $l$ - общее комплексное число, то оператор $L M_{l}$ имеет степень $l+m+1$ и длину $2 m+1$ (лемма 4.4). Следовательно, в силу леммы 4.3

$$
L M_{l}=C_{l} \frac{\phi(x)}{\phi(x+l+m+1)} T_{l+m+1}+\cdots
$$


с точностью до членов более низкой степени. Здесь $C_{l}$ возникает из коэффициента при $T_{l+m}$ в $M_{l}: A_{l+m}^{l}(x)=C_{l} \phi(x) / \phi(x+l+m)$. Отсюда вытекает, что, вычитая подходящее кратное оператора $M_{l+1}$ из $L M_{l}$, мы получаем оператор степени $\leqslant l+m-1$. Аналогично, $L M_{l}=S L M_{-l} S=$ $C_{-l} \phi(-x) / \phi(-x-l+m+1) T_{l-m-1}$ плюс члены более высокой степени, и мы можем сделать коэффициент при $T_{l-m-1}$ равным нулю, вычтя кратное оператора $M_{l-1}$. В результате мы получаем, что

$$
L M_{l}-\frac{C_{l}}{C_{l+1}} M_{l+1}-\frac{C_{-l}}{C_{-l+1}} M_{l-1}
$$

является оператором длины $<2 m$, коммутирующим с $L$, и по лемме 4.5 равен нулю. Отношения функций $C_{l}$ можно легко вычислить с помощью явных выражений для $A_{l+m}^{l}$, и они дают требуемый результат.

Таким образом, $M_{l}$, рассматриваемый как функция от $l$, является собственным вектором q-оператора Ламе в пространстве разностных операторов с «собственным значением» $L$.

Лемма 5.2. Пусть $\omega=1 / \gamma$. Тогда $M_{l+\omega}=M_{l} T_{\omega}$.

ДокАЗАТЕЛьство. Мы имеем

$$
M_{l+\omega}=\sum_{k=0}^{m} A_{l+\omega-m+2 k}^{l+\omega} T_{l+\omega-m+2 k}=\sum_{k=0}^{m} A_{l-m+2 k}^{l} T_{l-m+2 k} T_{\omega},
$$

поскольку коэффициент $A_{l-m+2 k}^{l}$ является $\omega$-периодическим как функция от $l$.

ПРЕДЛОЖЕНИЕ 5.3. Имеем

$$
M_{l} \psi(t, x)=\varepsilon_{l}(t) \psi(t, x),
$$

где собственное значение $\varepsilon_{l}(t)$ равно

$$
\frac{[2 m] ! \psi(t, l)}{[m] ! \psi(t, m)}
$$

ДокАЗАтЕЛЬСтво. Пусть $\psi(t, x, l)=M_{l} \psi(t, x)$, и обозначим через $\widehat{L}$ $q$-оператор Ламе, действующий на переменной $l$. Тогда

$$
\begin{aligned}
\widehat{L} \psi(t, x, l) & =\frac{[l+m]}{[l]} M_{l-1} \psi(t, x)+\frac{[l-m]}{[l]} M_{l+1} \psi(t, x) \\
& =L M_{l} \psi(t, x)=M_{l} L \psi(t, x)=\varepsilon_{L}(t) M_{l} \psi(t, x) .
\end{aligned}
$$

Пусть $\omega=1 / \gamma$. Пусть $e^{c}$ - мультипликатор функции $\psi(t, x): \psi(t, x+\omega)=$ $e^{c} \psi(t, x)$. Тогда, согласно лемме $5.2, \psi(t, x, l+\omega)=e^{c} \psi(t, x, l)$. Иными словами, $\psi(t, x, l)$ и как функция от $x$, и как функция от $l$, является собственной функцией оператора $L$ с одним и тем же собственным значением и с одним и тем же мультипликатором. Если $t$ находится в общем положении, то с точностью до нормализации существует только одна такая собственная функция. Значит,

$$
\psi(t, x, l)=f(t) \psi(t, l) \psi(t, x)
$$


для некоторой функции $f(t)$. С другой стороны, мы знаем, что если $l=m$, то $M_{m}$ равен тождественному оператору, умноженному на $[2 m] ! /[m] !$ Э. Это определяет $f$, и мы получаем

$$
\psi(t, x, l)=\frac{[2 m] !}{[m] !} \frac{\psi(t, l) \psi(t, x)}{\psi(t, m)} .
$$

В качестве следствия мы получаем, что соотношение из теоремы 1.3 является частным случаем более общих правил умножения:

СледСтвиЕ 5.4. Для общих $l, m \in \mathbb{C}$

$$
M_{l} M_{k}=\sum_{j} A_{j}^{l}(k) M_{k+j}
$$

где $A_{j}^{l}, j=l-m, l-m+2, \ldots, l+m,-$ коэффичиенты, определенные в $4_{\text {. }}$.

ДокАЗАТЕльСТво. В силу леммы 3.4 достаточно доказать это тождество для собственных значений $\varepsilon_{l}(t)$. Прежде всего, отметим, что предложение 5.3 может быть переписано после замены $x$ на $k$ в следующем виде:

$$
\sum_{j} A_{j}^{l}(k) \psi(t, k+j)=\varepsilon_{l}(t) \psi(t, k) .
$$

Значит,

$$
\begin{aligned}
\sum_{j} A_{j}^{l}(k) \varepsilon_{k+j}(t) & =\frac{[2 m] !}{[m] !} \sum_{j} A_{j}^{l}(k) \frac{\psi(t, k+j)}{\psi(t, m)} \\
& =\frac{[2 m] !}{[m] !} \varepsilon_{l}(t) \frac{\psi(t, k)}{\psi(t, m)}=\varepsilon_{l}(t) \varepsilon_{k}(t) .
\end{aligned}
$$

СлЕДСТВИЕ 5.5. Пусть $N=M_{m+1}-M_{-m-1}$. Тогда

$$
N \psi(t, x)=\varepsilon_{N}(t) \psi(t, x),
$$

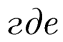

$$
\varepsilon_{N}(t)=\frac{[2 m] !}{[m] !}\left(e^{\gamma c} \prod_{j=1}^{m} \frac{\left[m+t_{j}+1\right]}{\left[m+t_{j}\right]}-e^{-\gamma c} \prod_{j=1}^{m} \frac{\left[m-t_{j}+1\right]}{\left[m-t_{j}\right]}\right), \quad e^{\gamma c}=\sqrt{b_{j}(t)} .
$$

Отображение $t \mapsto\left(\varepsilon_{L}(t), \varepsilon_{N}(t)\right)$ определяет бирациональный изоморфизм из двулистного накрытия $Y_{2}$ кривой Эрмита-Бете на кривую $\left\{(X, Y) \in \mathbb{C}^{2}\right.$ $\left.Y^{2}=P\left(X^{2}\right)\right\}$.

ДокАЗАТЕльСТво. Выражение для собственного значения взято из предложения 5.3.

По построению функция $\varepsilon_{L}(t)$ задает рациональное двукратное отображение из замыкания накрытия $Y_{2}$ на $\mathbb{P}^{1}$. Две точки в $\varepsilon_{L}^{-1}(p)$, где $p \in \mathbb{P}^{1}$ - точка в общем положении, связаны симметрией $(t, c) \mapsto(-t,-c)$. Собственное значение $\varepsilon_{N}$ является нечетным относительно этой симметрии, и мы имеем взаимно однозначное (в точках общего положения) отображение из $Y_{2}$ на указанную гиперэллиптическую кривую. 
ЗАмечАния. 1. Гиперэллиптическая кривая $Y^{2}=P\left(X^{2}\right)$ имеет на бесконечности двойную точку, которая распадается на две точки $P_{+}$и $P_{-}$двулистного накрытия $Y_{2}$ кривой Бете-Эрмита. Это две точки, в которых собственные значения наших коммутирующих операторов имеют полюсы. Полученные результаты можно рассматривать как вырожденный случай разностной версии конструкции Кричевера: Кричевер [5] ставит в соответствие гладкой проективной кривой $C$ рода $g$, двум ее точкам $P_{+}, P_{-}$и эффективному дивизору в общем положении $D$ степени $g$ функцию БейкераАхиезера $\psi(p, x)$, подходящим образом нормированную мероморфную функцию от $p \in C$ и $x \in \mathbb{Z}$ с дивизором (формальной суммой взятых с их кратностями полюсов минус взятые с их кратностями нули) $x\left(P_{+}-P_{-}\right)+D$. C каждой мероморфной функцией $f$ на $C$, которая регулярна на $C-\left\{P_{+}, P_{-}\right\}$, связан разностный оператор $M_{f}$ с целочисленными шагами, такой, что $\psi(p, \cdot)$ является его собственной функцией с собственным значением $f(p)$ и $f \mapsto M_{f}$ - гомоморфизм алгебр. В частности, если $P$ - полином нечетной степени $2 m+1$ без кратных корней, то кривая $Y^{2}=P\left(X^{2}\right)$ может быть компактифицирована присоединением двух точек $P_{+}, P_{-}$на бесконечности, причем полученная компактификация является гладкой гиперэллиптической кривой, и посредством выбора дивизора мы получаем некоторую пару коммутирующих разностных операторов $M_{X}$ и $M_{Y}$. Отметим, что, вообще говоря, функция Бейкера-Ахиезера выражается через тета-функцию Римана этой гиперэллиптической кривой, в то время как в данном случае ее можно выразить с помощью лишь некоторой эллиптической кривой.

2. Отметим аналогию с «алгеброй слияний». Пусть $N_{j k}^{i}=\operatorname{dim} \operatorname{Hom}\left(V_{i}, V_{j} \otimes\right.$ $\left.V_{k}\right)$ - размерности пространства гомоморфизмов, скажем, представлений простой группы Ли $G$. Здесь $V_{i}$ - неприводимые конечномерные представления, индексированные своими старшими весами $i \in P^{+}$. Введем разностные операторы $M_{l}$, действующие на функциях на $P^{+}$по формуле $M_{l} f(i)=$ $\sum_{j} N_{l j}^{i} f(j)$. Из коммутативности и ассоциативности тензорного произведения следует, что операторы $M_{l}$ коммутируют между собой. Кроме того, $M_{l} M_{k}=\sum_{j} N_{l k}^{j} M_{j}$ (см. следствие 5.4). Пусть $\psi(t, j)=\operatorname{tr}_{V_{j}}(t)$ - характер представления $V_{j}$. Это функция от $t$ из $T / W$, фактора картановского тора по группе Вейля. Тогда $\psi(t, j)$ как функция от $j$ при фиксированном $t$ является общей собственной функцией всех операторов $M_{l}$ :

$$
M_{l} \psi(t, \cdot)=\varepsilon_{l}(t) \psi(t, \cdot), \quad \varepsilon_{l}(t)=\frac{[2 m] ! \psi(t, l)}{[m] ! \psi(t, m)}
$$

(ср. предложение 5.3). Если мы заменим $G$ квантовой группой в корне из единицы, то применяется та же формула, за исключением того, что $P^{+}$заменяется некоторым конечным подмножеством, а $T$ - множеством точек конечного порядка $N$, зависящего от порядка этого корня из единицы, из картановского тора. В этом случае $\psi(t, j)$ обладает замечательной интерпретацией в терминах представлений группы $S L(2, \mathbb{Z})$, найденной Э. Верлинде [11], в контексте конформной теории поля. Имеется ли подобная интерпретация в нашем случае? 


\section{§6. Группа Галуа}

Вычислим «разностную группу Галуа» $q$-уравнения Ламе. Эта группа является разностным аналогом дифференциальной группы Галуа дифференциального уравнения. Стимулом для этого вычисления послужил недавний результат Бравермана, Этингофа и Гейтсгори [1], которые в случае дифференциальных уравнений охарактеризовали алгебраическую интегрируемость абелевостью соответствующей группы Галуа.

ОПРеделЕниЕ. Разностное поле - это поле $F$ вместе с некоторым его автоморфизмом $T \in \operatorname{Aut}(F)$. Расширением $E \subset F$ разностных полей называется подполе $E$ разностного поля $F$, такое, что $T(E) \subset E$. Автоморфизм разностного поля $F$ - это автоморфизм поля $F$, коммутирующий с $T$. Груnпой Галуа расширения $E \subset F$ разностных полей называется группа таких автоморфизмов поля $F$, ограничения которых на $E$ являются тождественным автоморфизмом.

Рассмотрим теперь наше разностное уравнение $L \psi=\varepsilon \psi$ при фиксированном общем $\varepsilon$. Произведя замену переменных $\psi(x)=u(x) \prod_{j=1}^{m}[x-j]$, мы придем к уравнению

$$
u(x+1)+\frac{[x+m][x-m-1]}{[x][x-1]} u(x-1)=\varepsilon u(x) .
$$

Коэффициенты этого разностного уравнения лежат в поле $E$ эллиптических функций с периодами $\omega, \omega^{\prime}$. Поле $E$ является разностным полем, в котором автоморфизм $T$ есть сдвиг: $T f(x)=f(x+1)$.

Пусть $F$ - дифференциальное поле, порожденное над $E$ мероморфными решениями разностного уравнения (5). Оно является полем всех рациональных функций от этих решений и их образов при $T^{j}, j \in \mathbb{Z}$, с коэффициентами из $E$.

Из теоремы 3.1 вытекает, что решения уравнения (5) суть линейные комбинации функций $u_{+}(x)$ и $u_{-}(x)=u_{+}(-x)$ с коэффициентами из $K$, поля мероморфных 1-периодических функций. Решение $u_{+}$имеет вид

$$
u_{+}(x)=e^{c \gamma x} \prod_{j=1}^{m} \frac{\left[x+t_{j}\right]}{[x-j]} .
$$

ТеОРема 6.1. Пусть $\gamma \in \mathbb{R}-\mathbb{Q} u \varepsilon-$ иисло в общем положении. Тогда группа Галуа расширения $E \subset F$ разностных полей изоморфна группе $K^{\times}$ ненулевых мероморфных 1-периодических функиий на комплексной плоскости. Функиия $h \in K^{\times}$соответствует автоморфизму, действующему на решениях по правилу $u_{ \pm}(x) \mapsto h(x)^{ \pm 1} u_{ \pm}(x)$.

Для доказательства этой теоремы нам нужны некоторые вспомогательные результаты.

ЛЕмма 6.2. Если $\Phi$ - некоторый элемент группь Галуа, то $\Phi\left(u_{ \pm}\right)=$ $h^{ \pm 1} u_{ \pm}$для некоторой функиии $h \in K^{\times}$. 
ДокАЗАТЕльство. Функция $u_{+}(x)$ умножается на постоянные множители, когда $x$ сдвигается на $\omega$ или $\omega^{\prime}$. Таким образом, $T u_{+} / u_{+}$- эллиптическая функция. Значит, если автоморфизм Галуа отображает $u_{+}$в функцию $\tilde{u}_{+}$, то $T \tilde{u}_{+} / \tilde{u}_{+}=T u_{+} / u_{+}$. Поэтому $\tilde{u}_{+}=h u_{+}$для некоторой функции $h \in K^{\times}$.

Аналогично, $u_{+}(x) u_{-}(x)$ - эллиптическая функция. Таким образом, если $u_{+}$отображается в $h u_{+}$, то $u_{-}$отображается в $h^{-1} u_{-}$.

Пусть $\widehat{E}$ - поле, порожденное полями $K$ и $E$.

ЛЕмма 6.3. $F=\widehat{E}\left(u_{+}\right)$, m. е. поле $F$ состоит из рачиональньх функиий от $u_{+}$скоэффициентами из $\widehat{E}$.

ДокАЗАтЕльство. Прежде всего заметим, что $K \subset F:$ элемент $h \in K$ есть отношение решений $h u_{+} / u_{+}$. Остается показать, что каждый элемент из $F$ можно записать как рациональную функцию от $u_{+}$с коэффициентами из $\widehat{E}$. Но доказательство предыдущей леммы показывает, что $u_{-}$и $T u_{+}$, a значит, и все решения, так же как и все их образы при $T^{j}, j \in \mathbb{Z}$, являются рациональными функциями от $u_{+}$с коэффициентами из $\widehat{E}$.

ЛЕмма 6.4. Функция $u_{+}$трансцендентна над $\widehat{E}$.

ДокАЗАтЕльство. Это означает, что $u_{+}$не является решением ни для какого полиномиального уравнения с коэффициентами из $\widehat{E}$. Допустим, что существует такое уравнение

$$
P\left(x, u_{+}(x)\right)=\sum A_{j}(x) u_{+}(x)^{j}=0, \quad A_{j} \in \widehat{E} .
$$

Tак как $\omega$ вещественно, то существует строго возрастающая последовательность целых чисел $n_{1}, n_{2}, \ldots$, такая, что расстояние между $n_{l} \omega$ и целочисленной решеткой сходится к нулю. Пусть $x$ - любое общее комплексное число, и положим $x_{l}=x+n_{l} \omega$. Тогда $\lim _{l \rightarrow \infty} A_{j}\left(x_{l}\right)=A_{j}(x)$ для всех $j$. $\mathrm{C}$ другой стороны, $u_{+}\left(x_{l}\right)=C^{n_{l}} u_{+}(x)$ для некоторой нетривиальной константы $C$. Поскольку $P\left(x_{l}, u_{+}\left(x_{l}\right)\right)=0$ для всех $l$, все коэффициенты $A_{j}(x)$ обращаются в нуль в $x$. Но $x$ произвольно. Поэтому все эти коэффициенты равны нулю тождественно. Противоречие.

Теперь доказательство теоремы 6.1 можно завершить. Осталось доказать, что для любой функции $h \in K^{\times}$существует единственный автоморфизм Галуа, переводящий $u_{+}$в $h u_{+}$.

Единственность вытекает из леммы 6.2 и леммы 6.3.

Чтобы доказать существование, мы должны показать, что для всех рациональных функций $f \in \widehat{E}(X)$ от одной переменной отображение

$$
f\left(u_{+}\right) \mapsto f\left(h u_{+}\right)
$$

определено корректно, т.е. не зависит от выбора функции $f$, используемой для представления элемента $f\left(u_{+}\right) \in F$, и что оно определяет автоморфизм разностных полей.

Отображение определено корректно: если $f\left(u_{+}\right)=g\left(u_{+}\right)$для рациональных функций $f=p / q, g=r / s$, то $u_{+}$является решением полиномиального 
уравнения $p s-q r=0$. В силу леммы 6.4 это уравнение должно быть тривиальным, а это означает, что $f=g$ в $\widehat{E}(X)$. В частности, $f\left(h u_{+}\right)=g\left(h u_{+}\right)$.

Ясно, что (6) определяет автоморфизм полей с обратным автоморфизмом $f\left(u_{+}\right) \mapsto f\left(u_{+} / h\right)$. Покажем, что он является автоморфизмом разностных полей. Мы имеем $T f\left(u_{+}\right)=\bar{f}\left(u_{+}\right)$. Рациональная функция $\bar{f}(X)$ имеет вид $(T f)(a X)$, где $a=T u_{+} / u_{+} \in E$ и $T f$ получается из $f$ применением автоморфизма $T$ к коэффициентам. Мы должны показать, что $T\left(f\left(h u_{+}\right)\right)=\bar{f}\left(h u_{+}\right)$. Но так как $T\left(h u_{+}\right)=h T\left(u_{+}\right)$, то

$$
T\left(f\left(h u_{+}\right)\right)=(T f)\left(h T\left(u_{+}\right)\right)=(T f)\left(h a u_{+}\right)=\bar{f}\left(h u_{+}\right) .
$$

Доказательство завершено.

ЗАмЕчАниЕ. Наша конструкция - это частный случай более общей конструкции для большей размерности: разностное поле размерности $n$ - это поле $F$ вместе с $n$ коммутирующими автоморфизмами $T_{1}, \ldots, T_{n}$. Определения расширений и групп Галуа обобщаются на случай произвольного $n$ очевидным образом. Пусть $F$ - поле функций $f\left(x_{1}, \ldots, x_{n}\right)$ и $T_{i}$ - операторы сдвига,

$$
T_{i} f\left(x_{1}, \ldots, x_{n}\right)=f\left(x_{1}, \ldots, x_{i}+a_{i}, \ldots, x_{n}\right), \quad i=1, \ldots, n .
$$

Скажем, что функция $g$ әлементарна относительно разностного поля $F$, если существуют функции $f_{1}, \ldots, f_{n}$ из $F$, такие, что $g$ удовлетворяет уравнениям

$$
T_{i} g / g=f_{i}, \quad i=1, \ldots, n \text {. }
$$

Пусть

$$
L_{j} f\left(x_{1}, \ldots, x_{n}\right)=0, \quad j=1, \ldots, k,
$$

- система линейных разностных уравнений с коэффициентами из разностного поля $F$. Предположим, что пространство ее решений имеет базис, состоящий из элементарных функций. Тогда группа Галуа этой системы является абелевой.

\section{ЛитературА}

1. Braverman A., Etingof P., Gaitsgory D. Differential Galois Groups and Algebraic Integrability of Quantum Integrable Systems. Alg-geom/9607012 (preprint).

2. Chalykh O., Veselov A. Commutative rings of differential operators and Lie algebras. Commun. Math. Phys., 126, 597-611 (1990).

3. Дубровин Б. А., Матвеев В. Б., Новиков С. П. Нелинейные уравнения типа Кортевега-де Фриза, конечнозонные линейные операторы и абелевы многообразия. УМН, 31, 55-136 (1976).

4. Felder G., Varchenko A. Algebraic Bethe ansatz for the elliptic quantum group $E_{\tau, \eta}\left(s l_{2}\right)$. Nucl. Phys. B, 480, 485-503 (1996).

5. Кричевер И. М. Алгебраические кривые и нелинейные разностные уравнения. УМН, 33, вып. 4, 215-216 (1978).

6. Кричевер И. М., Новиков С. П. Алгебры типа Вирасоро, римановы поверхности и структуры теории солитонов. Функц. анализ и прил., 21, вып. 2, 46-63 (1987). 
7. Krichever I., Zabrodin A. Spin Generalization of the Ruijsenaars-Schneider Model Non-Abelian 2D Toda Chain and Representations of Sklyanin Algebra, hep-th/9505039.

8. Mamford D. Algebro-geometric constraction of commuting operators and of solutions of the Toda latticeequation, Korteweg-de Vries equation andrelated non-linear equations. In: Proc. Intern. Symp. Algebraic Geometry, Kyoto, 1977. Kinokuniya Book Store, Tokyo, 1978, pp. 115-153.

9. Ruijsenaars S. N. M. Complete integrability of relativistic Calogero-Moser systems and elliptic function identities. Commun. Math. Phys., 110, 191-213 (1987).

10. Склянин E. K. О некоторых алгебраических структурах, связанных с уравнениями Янга-Бакстера. Представления квантовой алгебры. Функц. анализ и его прил., 17, вып. 4, 34-48 (1983).

11. Verlinde E. Fusion rules and modular transformations in $2 \mathrm{D}$ conformal field theory. Nucl. Phys. B, 300 [FS22], 360-376 (1987).

12. Whittaker E. T., Watson G. N. A Course of Modern Analysis, Cambridge University Press, 1915.

Centre Emile Borel, Institut Henri Poincaré,

Поступило в редакцию

Université Pierre et Marie Curie, France

10 октября 1997 г.

Department of Mathematics, University

of North Carolina at Chapel Hill, USA 